\title{
Reports of assistance with activities of daily living: Advanced cancer patient \& spouse caregiver concordance
}

\author{
Amy K. Otto, PhD; Dana Ketcher, PhD, MPH, CPH; Maija Reblin, PhD \\ Moffitt Cancer Center, Tampa, Florida
}

$\underset{\text { CANGER CENTER }}{\text { MOP. }}$

\section{Introduction}

- Little research exists on the agreement between cancer patients and proxies about patient functional status and caregiver assistance with activities of daily living (ADLs) and instrumental activities of daily living (IADLs)

- Most existing work focuses on dementia patients or older adults, or on proxies other than family caregivers (e.g., medical providers)

- Aims:

1. Describe patient-caregiver concordance of reported ADLs and IADLs in the context of advanced cancer

2. Identify factors associated with lower patient-caregiver concordance

\section{Method}

- Participants: 88 adult patients with stage III-IV GI or thoracic cancer (PTs) and their opposite-sex spouse caregivers (CGs)

- Procedure: Participants completed a cross-sectional questionnaire assessing:

- Caregiver assistance to patients in ADLs/IADLs: yes/no items about 8 ADLs and 7 IADLs

- Relationship satisfaction: 4-item Couples Satisfaction Index (CSI-4)

- Caregiver burden (caregivers only): 14-item Caregiver Burden Scale (CBS)

Results

Table 1. Demographics and descriptive statistics

\begin{tabular}{|c|c|c|c|c|}
\hline & \multicolumn{2}{|c|}{ PTs } & \multicolumn{2}{|c|}{ CGs } \\
\hline & $M$ & $S D$ & $M$ & $S D$ \\
\hline Age & 66.8 & 9.2 & 64.7 & 9.2 \\
\hline Years married & 34.1 & 15.6 & 34.1 & 15.9 \\
\hline Relationship satisfaction & 20.5 & 3.1 & 19.8 & 4.0 \\
\hline \multicolumn{5}{|l|}{ Caregiver burden } \\
\hline Objective burden & -- & -- & 22.1 & 3.7 \\
\hline Stress burden & -- & -- & 14.1 & 2.5 \\
\hline \multirow[t]{2}{*}{ Demand burden } & -- & -- & 11.8 & 3.0 \\
\hline & $n$ & $\%$ & $n$ & $\%$ \\
\hline Female & 25 & 28.4 & 63 & 71.6 \\
\hline Non-Hispanic & 85 & 96.6 & 81 & 92.0 \\
\hline \multicolumn{5}{|l|}{ Race } \\
\hline White & 82 & 93.2 & 79 & 89.8 \\
\hline Black & 5 & 5.7 & 4 & 4.5 \\
\hline Other & 1 & 1.1 & 3 & 3.4 \\
\hline \multicolumn{5}{|l|}{ Education } \\
\hline$\leq$ High school & 16 & 18.2 & 16 & 17.6 \\
\hline $\begin{array}{l}\text { Vocational school or some } \\
\text { college }\end{array}$ & 29 & 33.0 & 32 & 36.4 \\
\hline College (4 years) & 16 & 18.2 & 13 & 14.8 \\
\hline$>$ College & 27 & 30.7 & 27 & 30.7 \\
\hline \multicolumn{5}{|l|}{ Employment } \\
\hline Not employed & 67 & 76.1 & 53 & 60.2 \\
\hline Employed part-time & 5 & 5.7 & 11 & 12.5 \\
\hline Employed full-time & 15 & 17.0 & 21 & 23.9 \\
\hline \multicolumn{5}{|l|}{ Household income } \\
\hline$\$ 10,000-\$ 24,999$ & 5 & 5.7 & 4 & 4.5 \\
\hline$\$ 25,000-\$ 39,999$ & 13 & 14.8 & 14 & 15.9 \\
\hline$\$ 40,000-\$ 49,999$ & 8 & 9.1 & 8 & 9.1 \\
\hline$\$ 50,000-\$ 74,999$ & 27 & 30.7 & 24 & 27.3 \\
\hline$\geq \$ 75,000$ & 33 & 37.5 & 37 & 42.0 \\
\hline
\end{tabular}

Table 2. Assistance to patient in each ADL/IADL

\begin{tabular}{|c|c|c|c|c|c|}
\hline \multirow{2}{*}{ Activity } & \multicolumn{2}{|c|}{$\begin{array}{l}\text { \% Reporting PT } \\
\text { Gets Assistance }\end{array}$} & \multicolumn{3}{|c|}{ PT-CG Disagreement } \\
\hline & PT & CG & $r$ & $\begin{array}{l}\text { \% Dyads that } \\
\text { Disagreed }\end{array}$ & $\mathbf{K}$ \\
\hline \multicolumn{6}{|l|}{ ADLs } \\
\hline Feeding & 6 & 9 & $.44^{\star \star \star}$ & 8 & .42 \\
\hline Toileting & 3 & 3 & $.66^{\star \star \star}$ & 2 & .66 \\
\hline Selecting proper attire & 14 & 12 & $.39^{\star \star \star}$ & 15 & .39 \\
\hline Grooming & 8 & 12 & $.52^{\star \star \star}$ & 9 & .51 \\
\hline Continence maintenance & 2 & 3 & $.62^{\star \star \star *}$ & 3 & .56 \\
\hline Dressing & 9 & 13 & $.60^{\star \star \star}$ & 8 & .59 \\
\hline Bathing & 8 & 5 & $.74^{\star \star *}$ & 3 & .71 \\
\hline Walking/transferring & 9 & 11 & $.63^{\star * *}$ & 7 & .63 \\
\hline None & 76 & 69 & $.26^{*}$ & 30 & .26 \\
\hline \multicolumn{6}{|l|}{ IADLs } \\
\hline Managing finances & 56 & 61 & $.56^{\star * *}$ & 22 & .56 \\
\hline Transportation & 51 & 58 & $.55^{\star \star *}$ & 23 & .54 \\
\hline Shopping & 69 & 70 & .11 & 37 & .11 \\
\hline Meal preparation & 76 & 77 & $.27^{*}$ & 26 & .27 \\
\hline Using phone & 18 & 25 & $.34^{* *}$ & 23 & .33 \\
\hline Medication management & 39 & 45 & $.59^{* \star *}$ & 20 & .58 \\
\hline Housework & 75 & 85 & .06 & 31 & .05 \\
\hline None & 11 & 7 & $.33^{\star \star}$ & 11 & .32 \\
\hline
\end{tabular}

- PT and CG reports of ADL assistance were positively correlated, but not all IADL reports were correlated

- Dyads disagreed more about IADLs $(24.2 \%, S D=20.7)$ than ADLs $(9.5 \%$, $S D=13.1)$

- CGs reported that PTs received assistance in more activities than PTs reported $(p=.042)$

- Older PT age was associated with greater ADL disagreement $(B=.008, p=$ .017)

- Greater CG stress burden $(B=-.011, p=.022)$ and greater PT relationship satisfaction $(B=-.009, p=.028)$ were associated with lower disagreement about activities

\section{Conclusions}

- Consistent with work in other populations, results suggest that patient-caregiver reports of patient independence in ADLs/IADLs are mostly correlated, but caregivers report that patients receive significantly more assistance than patients report receiving

- Results provide additional insight about patient-proxy ratings of functional status in the context of advanced cancer

- Findings suggest that the relationship context may influence perceptions of patient independence, and high disagreement may reflect "invisible support" from spouse-caregivers 